\title{
Optimized integrated design of a high-frequency medical ultrasound transducer with genetic algorithm
}

\author{
Ali Babazadeh Khameneh ${ }^{1} \cdot$ Hamid Reza Chabok ${ }^{2} \cdot$ Hossein Nejat Pishkenari $^{3}$ (i)
}

Received: 3 October 2020 / Accepted: 4 May 2021

Published online: 19 May 2021

(c) The Author(s) 2021 OPEN

\begin{abstract}
Designing efficient acoustic stack and elements for high-frequency (HF) medical ultrasound (US) transducers involves various interrelated parameters. So far, optimizing spatial resolution and acoustic field intensity simultaneously has been a daunting task in the area of HF medical US imaging. Here, we introduce optimized design for a 50-MHz US probe for skin tissue imaging. We have developed an efficient design and simulation approach using Krimholtz, Leedom and Matthaei (KLM) equivalent circuit model and spatial impulse response method by means of Field II software. These KLM model and Field II software are integrated, and a GA algorithm is used to optimize the design of the US transducer to obtain the best imaging performance. As a result, a $50-\mathrm{MHz}$ single element probe is effectively optimized with $5 \mathrm{~mm}$ acoustic focal length, $72 \mu \mathrm{m}$ lateral, and $42 \mu \mathrm{m}$ axial imaging resolution, with an enhancement in imaging resolution over the conventionally designed and simulated probe by $10 \%$. This work has the potential to benefit many applications that require a fast, high-resolution and strong US focus in skin imaging.
\end{abstract}

Keywords Medical ultrasonic transducers · Optimized design · Genetic algorithm

\begin{tabular}{|c|c|c|}
\hline \multicolumn{2}{|c|}{ List of symbols } & Piezo-thickness \\
\hline \multicolumn{2}{|c|}{ KLM Krimholtz, } & Acoustic impedance \\
\hline GA & Genetic algorithm & \\
\hline$P(z)$ & Sound pressure at a distance of $z$ & \\
\hline$a$ & Sound attenuation coefficient & 1 Introduction \\
\hline BW & Bandwidth & \\
\hline$\rho$ & Density & Creating a cross-sectional image of tissues using ultrasonic \\
\hline$c$ & Speed of sound & waves has become very common in modern medicine, due \\
\hline$\lambda$ & Wavelength of the sound & to being less expensive and risky as opposed to common \\
\hline PZT & Lead zirconate titanate & imaging methods [1]. The imaging resolution of ultrasonic \\
\hline PMN_PT & Lead magnesium niobate-lead titanate & imaging increases with increasing working frequency of \\
\hline $\mathrm{LiNbO} 3$ & Lithium niobate & the US probe, so HF US imaging has become a new and \\
\hline$F_{0}$ & Center frequency & valuable tool in applications such as skin imaging $[2-4]$ \\
\hline$n$ & Wave number & eye imaging $[1,5]$, intravascular imaging [6-9], Carotid \\
\hline
\end{tabular}

$\square$ Hamid Reza Chabok, chabok@usc.edu; Ali Babazadeh Khameneh, ali.babazadehkhameneh@mech.sharif.edu; Hossein Nejat Pishkenari, nejat@sharif.edu| ${ }^{1}$ Department of Mechanical Engineering, Sharif University of Technology, Tehran, Iran. ${ }^{2}$ Department of Industrial and Systems Engineering, University of Southern California, Los Angeles, CA, USA. ${ }^{3}$ Department of Mechanical Engineering, Sharif University of Technology, Tehran, Iran.

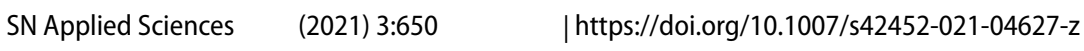


artery imaging and elastography $[10,11]$ and small animal imaging $[12,13]$.

Crossing the boundary of mediums with different acoustic properties, echo waves reflect from that boundary and are received by the transmitting probe and turned into voltage signals. By detecting the amplitude of these echoes, a line of black and white image can be produced regarding the strength of the reflection in the boundary. Then, by moving the single element probe in a line or changing the active elements in an array probe and repeating the same procedure, a cross-section image can be produced. This method is called brightness mode or B-mode imaging [14]. Single element piezoelectric probes can be designed in small geometries to enable the insertion of these elements using a catheter into the body to perform intravascular imaging [8], imaging the retina [15] and so on. US imaging can also be combined with other methods for imaging to bring about powerful imaging technics such as photoacoustics [16] and US shear wave elastography.

Non-imaging applications include particle manipulation and trapping, in which a pressure field is produced using a highly focused US probe that can move or trap particles [17-20], drug delivery [21], elastography of muscles and tissues [22] and high-intensity focused US (HIFU), which involves high energy US waves producing heat in specific point in tissue to destroy cells [23], increase of blood flow and applications like face lifting $[24,25]$ and pain management [26]. There are also many low-frequency applications such as ultrasonic scalps for surgery [27].

Acoustic impedance $(Z=\rho c)$ of the two adjacent mediums in a boundary determines the amount of ultrasonic wave that reflects or transmits through the boundary ( $\rho$ is the density and $\mathrm{c}$ is the speed of sound in the medium). The higher the acoustic impedance difference between two mediums, the more sound waves are reflected and less sound is transmitted through their boundary. This calls for a procedure named "acoustic matching" [28] in which one or two layers of specific materials are used to increase the transmission of sound waves from piezoelectric element into the tissue.

If the working frequencies are increased beyond 40 $\mathrm{MHz}$, micrometer resolutions can be obtained, as in [29] a frequency of $300 \mathrm{MHz}$ have resulted in $6.4 \mu \mathrm{m}$ imaging resolution. The downside is that increasing the frequency increases the attenuation of the sound waves in tissue as shown in Eq. 1 in which $\alpha$ is the attenuation coefficient and it increases with frequency; also $z$ is the distance and $P(z)$ is the pressure of the sound wave in the distance of $z$ [28].

$P(z)=P(0) e^{-\alpha z}$
This equation shows that $\mathrm{HF}$ imaging can be used for superficial tissues, and for deeper tissues one must use a needle to enter the US probe in the tissue to compensate for the lack of penetration.

In the literature [30], there are ways to calculate a rough estimation of the best possible imaging resolution for an US probe based on its geometry, focal distance (the distance of maximum sound pressure point in the medium to the probe) and bandwidth (which can be calculated from the frequency spectrum of resulting pressure or speed of vibration of the probe surface touching the tissue, when it is excited by an impulse voltage), as can be seen in the following equation, in which $R_{\text {axial }}$ and $R_{\text {lateral }}$ are resolutions in the axial and lateral direction of the probe, $c$ is the speed of sound in the medium, $B W$ is the $-6 \mathrm{~dB}$ bandwidth of the probe (in $\mathrm{Hz}$ ), $f$ number is defined as the ratio of the focal distance to head diameter of the probe, and $\lambda$ is the wave length of the sound wave in the medium [30-32]. Imaging resolution refers to the smallest length of tissue that can be detected by the probe and imaging process.

$R_{\text {axial }}=\frac{0.5 c}{B W}=\frac{0.5 c}{t(-6 \mathrm{~dB})}$

$R_{\text {lateral }}=f$ number $\cdot \lambda$

These two equations show that high bandwidth and low $f$-number and wavelength lead to better imaging resolutions.

To more accurately calculate the resulting resolutions and electrical behavior of a certain probe design, simulations need to be performed. In this paper, two different simulation methods for HF US probes are used together and used in an optimization loop in order to fine tune the design parameters of a probe. This probe will be designed for a medical imaging application.

Typical optimization methods for ultrasonic transducers depend upon the selected frequency. For lower-frequency transducers (up to $1 \mathrm{MHz}$ ), optimization mostly involves using FEM to solve and calculated the resulting vibrations of the transducer's head and the aim is to increase the amplitude of this vibration which is done either by the FEM softwares or Nimbus method [33-36]. But when the frequency is increased to the level suitable for medical purposes, the optimization process includes the process of changing the layers thickness or materials and manufacturing the transducer to measure the resulting electric signals or pressure fields [37]. FEM can still be used in this context and frequencies, but the required processing power and time are a lot. Instead of FEM, one can use simpler and more direct methods, such as KLM method [38] or Field II algorithm [39]. There are papers that have used the KLM method to calculate the resulting electrical signals 
and optimizing it by changing the thickness of the transducer layers [40] or the Field II methodology to enhance the pressure field characteristics of a transducer array [41].

It will be shown that the probe's characteristics can be optimized based on the resulting resolutions and the results will be compared to the initial design and also this optimization loop can be used to make a complete design and optimization procedure for any geometry, frequency, focal length and resolution requirements.

\section{Design requirements}

Considering the work done on HF US skin imaging and high benefits of HF US imaging in detecting different tumors, measurement of skin layer thicknesses, etc. [3, 42-44], this application was chosen to design this US probe for. In order to successfully detect and measure skin layer thicknesses and small tumors in the size of less than $0.2 \mathrm{~mm}$ in the depth of up to $6 \mathrm{~mm}$ of skin, the center frequency of $50 \mathrm{MHz}$ is a better choice $[2,42,45]$. Considering the required axial and lateral resolutions of less than 100 $\mu \mathrm{m}$ and using the theoretical values derived from Eqs. 2 and 3 , dimension of $3 \mathrm{~mm}$ in diameter and a focal point of $5 \mathrm{~mm}$ were chosen. This design would result in a 3 to 6 $\mathrm{mm}$ depth of penetration and theoretical resolutions better than $100 \mu \mathrm{m}$ which is suitable for this application.

\subsection{Materials and design}

Overall, a single element, HF US probe consists of a piezoelectric material layer, backing layer and matching layer (Fig. 1). All of these layers aim to produce a strong US wave, ensure its good transfer to the tissue and reduce the received disturbances.

Exciting the piezoelectric material with electrical pulses induces electrical field in it and results in strains that can produce sound waves. Different piezoelectric materials like PZT (lead zirconate titanate), PMN PT (lead magnesium niobate-lead titanate), LiNbO3 (lithium Niobate) or piezo-polymers have been used in the literature $[18,46]$. Here, lithium niobate was chosen because of its high sound speed, and high electromechanical coupling

Table 1 LiNbO3 piezoelectric properties (Boston Piezo-optic, Bellingham, MA)

\begin{tabular}{ll}
\hline Properties & LiNbO3 \\
\hline Electromechanical coupling coeff $(\mathrm{kt})$ & 0.49 \\
Density $\left(\mathrm{kg} / \mathrm{m}^{3}\right)$ & 4640 \\
Longitudinal sound velocity $(\mathrm{m} / \mathrm{s})$ & 7340 \\
Acoustic impedance $(\mathrm{MRayl})$ & 34.0 \\
Curie temperature $\left({ }^{\circ} \mathrm{C}\right)$ & 1150 \\
Relative clamped dielectric constant $(\varepsilon s / \varepsilon 0)$ & 39 \\
\hline
\end{tabular}

Fig. 1 Schematics of a single element HF US probe

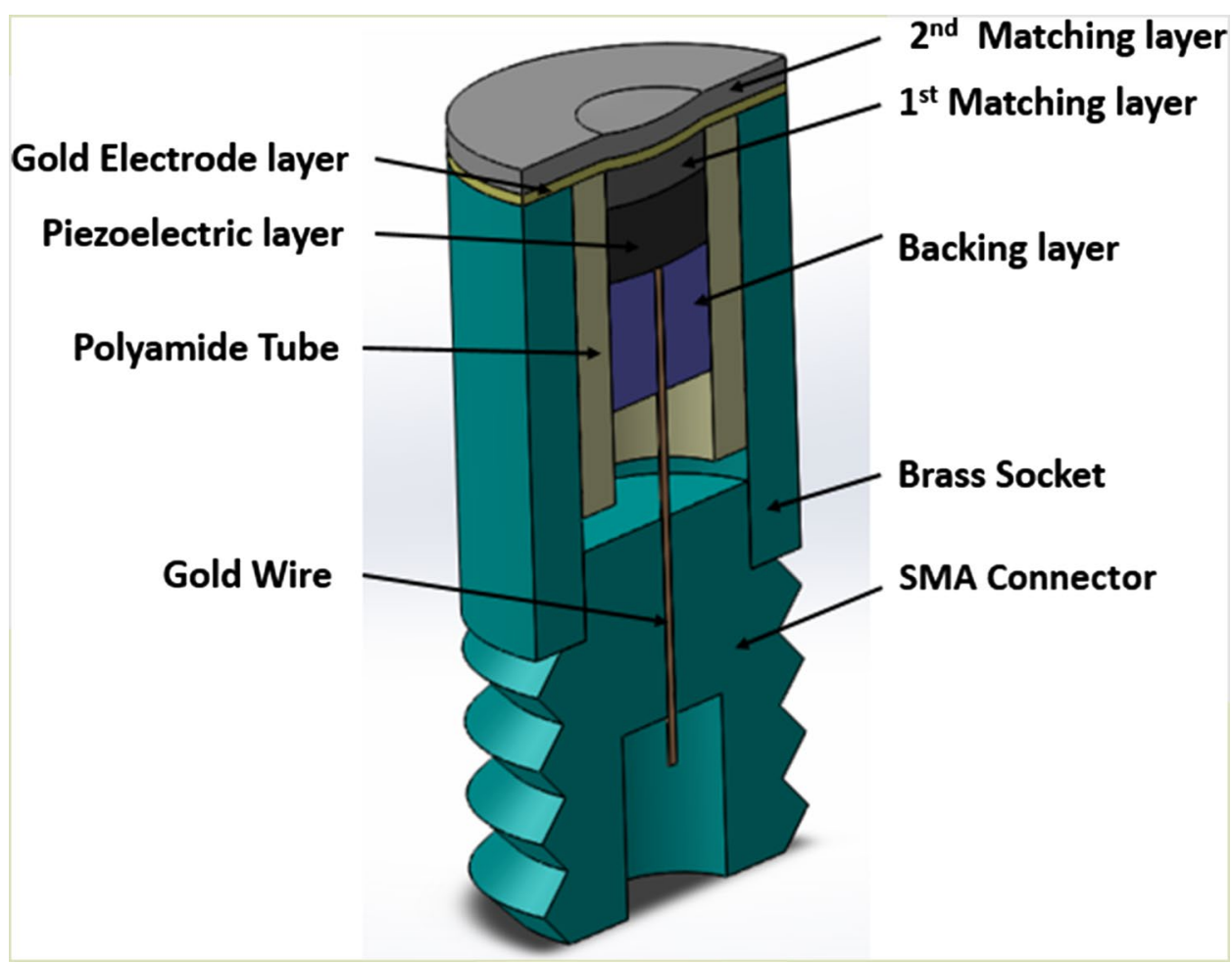


factor that results in a good energy conversion and better sensitivity. Its properties can be seen in Table 1. The piezoelectric material must be lapped to a thickness that will result in the theoretical center frequency of $50 \mathrm{MHz}$, which can be calculated from the following equation, in which $\mathrm{c}$ is the sound speed, $\mathrm{t}$ is the thickness of the layer, and $\mathrm{n}$ is the wave number [28].

$f_{0}=\frac{n c}{2 t}$

Equation 4 shows that the theoretical center frequency of a piezoelectric element depends on the sound speed in the material and inversely depends on the thickness of the element.

Behind the active layer, there is the backing layer which consists of large amounts of attenuating material in order to completely damp the sound waves produced by the active layer and propagated toward the back and it also does not let them reflect back toward the active layer. This results in a cleaner and clearer output signal.

Between the active layer and the tissue, there are one or two matching layers that have an intermediate acoustic impedance compared to the tissue and active layer. Since there is a huge difference in these impedances (1.5 MRayl for tissue and 38 MRayl for LiNbO3), these intermediate impedances were chosen in a way to increase the transmission of sound waves from active layer into the tissue by reducing this difference in steps. In the literature, the following values are suggested for the matching layer's acoustic impedance. $Z_{1}$ and $Z_{2}$ are the acoustic impedances of the piezo-material and the medium, respectively [28].

$Z=\frac{P}{U_{z}}=\rho c$

$Z_{m 1}=\left(Z_{1}^{4} Z_{2}^{3}\right)^{\frac{1}{7}}$

$Z_{m 2}=\left(Z_{1} Z_{2}^{6}\right)^{\frac{1}{7}}$

Equation 5 shows that acoustic impedance is defined as the ratio of the sound pressure in a medium to the speed of particles in the medium. $Z_{m 1}$ and $Z_{m 2}$ show the theoretical impedance for the two matching layers for the best transmission of sound wave.

In theory, a thickness of $\lambda / 4$ is recommended for each layer [47] ( $\lambda$ is the wave length of sound in each layer) for the best transmission of waves. The materials chosen and their properties can be seen in Table 2 , in which the values were extracted from $[46,48]$.

The natural focal point of an unfocused probe can be calculated with the Fresnel Limit [49]:

Fresnel Limit $=\frac{a^{2}}{\lambda}[\mathrm{m}]$

In the above equation, $a$ is the radius of the head and $\lambda$ is the wavelength of the sound in the medium. Considering the center frequency of $50 \mathrm{MHz}$ and the head dimension of $3 \mathrm{~mm}$, the Fresnel limit would be $75 \mathrm{~mm}$, which is far from the desired focal length of $5 \mathrm{~mm}$. For obtaining this short focal length, a procedure called "press focusing" has been proposed in the literature [50] to press the head surface with a ball bearing at special temperature conditions to focus the probe to the desired focal length.

First matching layer is silver epoxy, which is conductive and can be used to form the ground connection to the piezo. Its acoustic impedance is close to the value derived from Eq. 6 for said frequency and piezoelectric material, which is $Z_{1}=8.95$ MRayl. Parylene $C$ was chosen as the second matching layer since it has acoustic impedance near the preferred value of $Z_{2}=2.34 \mathrm{MRayl}$ from Eq. 7, and also, it is a standard coating material for medical equipment. As it can be seen, the acoustic impedances of the chosen materials are not exactly as calculated using the mentioned equations above for matching layer impedances. This means that the thicknesses of the layers must be optimized instead of using the theoretical values of $\lambda / 4$ to obtain better results.

\subsection{Simulation methods}

Simulating HF US probes involves two stages. First the probe's response to input voltage excitation and its electrical characteristics must be simulated, which was done using equivalent electrical circuit methods. In this paper, KLM equivalent circuit was used for this stage. Using this
Table 2 Backing and matching material properties

\begin{tabular}{lllll}
\hline Material & $\begin{array}{l}\text { Long velocity } \\
(\mathrm{m} / \mathrm{s})\end{array}$ & Density $\left(\mathrm{kg} / \mathrm{m}^{3}\right)$ & $\begin{array}{l}\text { Impedance } \\
\text { MRayl }\end{array}$ & Loss $\mathrm{dB} / \mathrm{mm}$ \\
\hline E-Solder 3022 & 1850 & 3200 & 5.92 & 110 \\
2-3 Micron silver epoxy & 1900 & 3860 & 7.33 & 13.8 \\
Parylene C & 2350 & 1180 & 2.77 & 0.56 \\
\hline
\end{tabular}

Values extracted from $[46,48]$ 
model, the resulting vibrations at the probe's head produced from the input electrical excitation at the probe's terminal is simulated. Second the resulting pressure fields in a single medium and in a medium with attenuating tissues must be simulated. These pressure fields result from the vibration at the surface of the medium coming from the probe (which was simulated before using KLM model). This simulation can be done using either finite element methods or using a simplified model to obtain analytical answers. In this paper, Field II code using analytical methods was used to simulate pressure fields and B-mode imaging to determine the resolution of the probe.

For calculating the pressure field in the medium, the spatial impulse response method expressed in [51-53] was used at [54-56] to develop a source code called Field II that can calculate the pressure field for different geometry and input voltage to acceleration profile. This method uses simplifying assumptions such as small amplitude of resulting pressures and limited medium (which are valid in medical US) to linearize the nonlinear equations of sound propagation and then analytically calculates the resulting pressure in any point of medium in front of the probe for each surface element of it and then uses superposition to add them together. Also, this code can define points in the medium to have different attenuation and sound scattering properties to simulate any non-uniform medium for simulating the resulting pressure field and by receiving pressure waves at the probe's surface, one can simulate pulse-echo or B-mode imaging [39].

The KLM method (which is based upon [38]) uses equivalent electrical elements for each acoustic element, and this results in an electrical circuit in which current and voltage of each element are equivalent to vibration speed and force produced in each acoustic element, respectively. The input to this circuit is the same exciting voltage signal used to excite the probe, and the output is the force and speed of the element touching the medium or tissue [38]. Using matrix form, this method was coded into Matlab ${ }^{\mathrm{TM}}$ and used to simulate the probe. The inputs of the code are the geometry of the head, acoustic properties of all layer's materials and their thicknesses and working frequency. The outputs of the code are the force and speed of vibration at the end of the second matching layer.

To Attach the two methods together, a Matlab ${ }^{\mathrm{TM}}$ code was developed by the author to simulate the pressure field and also perform a B-mode imaging of very thin tungsten wires placed in the medium (which is chosen to be water). The diameter of the simulated wire was $20 \mu \mathrm{m}$ which is well under the resolution of the probe, so the resulting image would show the smallest image possible and therefore it can be used to determine the resolution of the probe. This procedure is called "Wire Phantom Imaging" and a schematic of the setup used in this test and its simulated results can be seen in Fig. 6 .

The material properties of the layers are used in the KLM method and so changing the layer thicknesses or materials will result in the change in head velocity of the probe. Since this velocity profile is directly used in Field II algorithm, the resulting pressure field will also change.

\subsection{Optimization procedure}

As it was mentioned before, the probe design based on the theoretical layer thicknesses and acoustic impedance value does not necessarily have the optimal sound transmission and resulting resolution. Also, the materials available for matching layers have a different acoustic impedance from the presented values, not to mention that the presented impedance in the literature is experimental by itself. This shows that optimization is required to have the best results.

By choosing the application, the head geometry and center frequency will be known. Considering the acoustic impedance values for the matching layers, closest materials are chosen for these layers.

If the surface geometry, the center frequency and the materials used in the probe are set, the variables of the design would be the thicknesses of the backing, active and matching layers. Since the backing only has the absorbing role, its thickness must be high enough to damp all the sound waves entering it and more than that does not affect the results (this length can be calculated considering the attenuation of the material and how long it should be to attenuate certain level of sound). So only the thicknesses of the active and matching layers must be calculated. These values are theoretically known (half wave length for the active layer and quarter wave length for matching layers), but since the matching layer materials cannot be chosen to have the exact acoustic impedance as suggested and also the suggestion values are experimental, an optimization can be done on these thicknesses to improve the resulting imaging resolutions.

The resulting resolution of a design can be calculated using the Field II source code using the phantom wire imaging as said above. So, an optimization loop can be created with the inputs of the thicknesses of the layers and the output of the resulting resolutions. First, the KLM model is used to calculate the acceleration of the second matching layer in response to an impulse excitation; then, this profile will be passed to the Field II code as an input and then using the wire phantom imaging simulation the resulting resolutions are calculated.

In this paper, genetic algorithm, which is one of the evolution-based optimization methods, was chosen to optimize the design. 
This paper intends to report a methodology that can be used to automate and optimize the previously mostly manual process of design of a transducer based on a given pressure field, which includes the intensity, the focal points and the transducer geometry to be built. To make such a pressure field, there are many parameters that can be changed such as layer thickness, center frequency, material choices, radius of the pressure field, focusing of the head, etc. To further advance the proposed algorithm, a specific application has been chosen and so the parameters' range has been constrained to achieve a more desirable acoustic pressure field. To find the Global minimum of the optimization with this many parameters, GA is among the best tools.

The GA used in this paper uses random values within the designated input parameters' range as a starting population [57]. The fitness function created for this algorithm is the sum of the dimensions of the $b$-mode imaging of a single wire phantom simulated for the designed probe in the Field II program. This fitness function can get more and more complicated if more characteristics of the probe are chosen for optimization. The initial values of genetic algorithm were set to be on the theoretical values derived from Eqs. 4 to 7. Every time genetic algorithm was used, these initial values were changed by a random value generated to change the starting point of the algorithm in a defined upper and lower bound. This ensures that the global minimum point is found [57]. The genetic algorithm had a population of the size between 200 and 400 and generations between 1000 and 3000 .

The overall optimization algorithm used can also be seen in the flowchart in Fig. 2. By choosing the application the minimum resolution, minimum penetration distance and focal distance will be extracted. With this information, the center frequency and head dimension and piezo-material are chosen and using the impedance equations for matching layers, the nearest materials and a suitable backing material are selected. This stage is also the beginning of the outer optimization loop in which materials, head dimension and center frequency are inputs of the optimization. Also, at this stage, theoretical resolution and penetration distance can be calculated and checked with the requirements. After this, the thicknesses of the layers are chosen and this is the beginning of the inner optimization loop, in which the inputs are the thicknesses of the layers. Next, the dimensions, frequency and materials are used in the KLM model to extract the electrical impedance plot and impulse response of the probe. This response is fed into the Field II code, and pressure field and simulated imaging resolutions are extracted. These resolutions are the outputs of the optimization loops, in which the thicknesses of the layers are optimized and if needed the center frequency and head dimensions are optimized as well. Both these loops can be done using genetic algorithm.

\section{Results and discussion}

Considering the theoretical values for the probe layers for the $50 \mathrm{MHz}$ probe with $3 \mathrm{~mm}$ head diameter, the following dimensions in Table 3 in the "not optimized" columns can be obtained for the probe. Using these values, the KLM and Field II simulations were performed and the results are shown in Figs. 3, 4, and 5.

The electrical impedance plot in Fig. 3 shows the probe's center frequency to be about $48 \mathrm{MHz}$, and the impulse response in Fig. 4 shows the $-6 \mathrm{~dB}$ bandwidth of $80 \%$ (38.4 $\mathrm{MHz}$ in the center frequency of $48 \mathrm{MHz}$ ). Also, the peakto-peak voltage of the impulse is seen to be $7.5 \mathrm{kPa}$. The simulated pressure field can be seen in Fig. 5 and the simulated b-mode image from four $20 \mu \mathrm{m}$ wires at around the

Fig. 2 Flowchart of the optimal design process

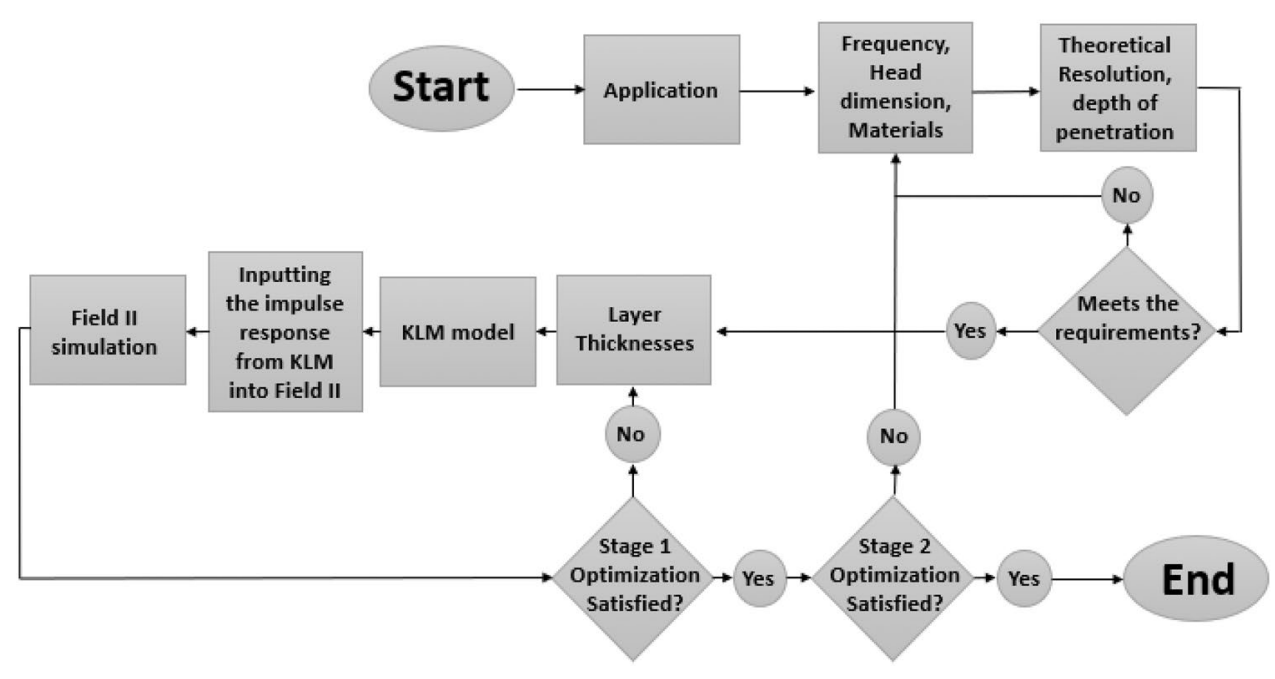


Table 3 Optimized probe layer thicknesses

$\begin{array}{llll}\text { Thickness optimized } & \begin{array}{l}\text { Thickness to sound wavelength } \\ \text { of the material. optimized }\end{array} & \begin{array}{l}\text { Thickness } \\ \text { unoptimized }\end{array} & \begin{array}{l}\text { Thickness in sound wave- } \\ \text { length of the material. } \\ \text { unoptimized }\end{array}\end{array}$

\begin{tabular}{lllll}
\hline E-Solder 3022 & $5.0 \mathrm{~mm}$ & 120 & $5 \mathrm{~mm}$ & 120 \\
LiNbO3 & $61.8 \mu \mathrm{m}$ & 0.490 & $73.6 \mu \mathrm{m}$ & 0.5 \\
2-3 Micron silver epoxy & $8.60 \mu \mathrm{m}$ & 0.226 & $9.5 \mu \mathrm{m}$ & 0.25 \\
Parylene & $10.80 \mu \mathrm{m}$ & 0.229 & $11.8 \mu \mathrm{m}$ & 0.25 \\
\hline
\end{tabular}

Fig. 3 The electrical impedance (blue) and its phase (red) of the probe with theoretical thicknesses resulting from the KLM model
Fig. 4 The pressure profile in response to impulse voltage resulting from the KLM model. The red is the frequency spectrum of the signal

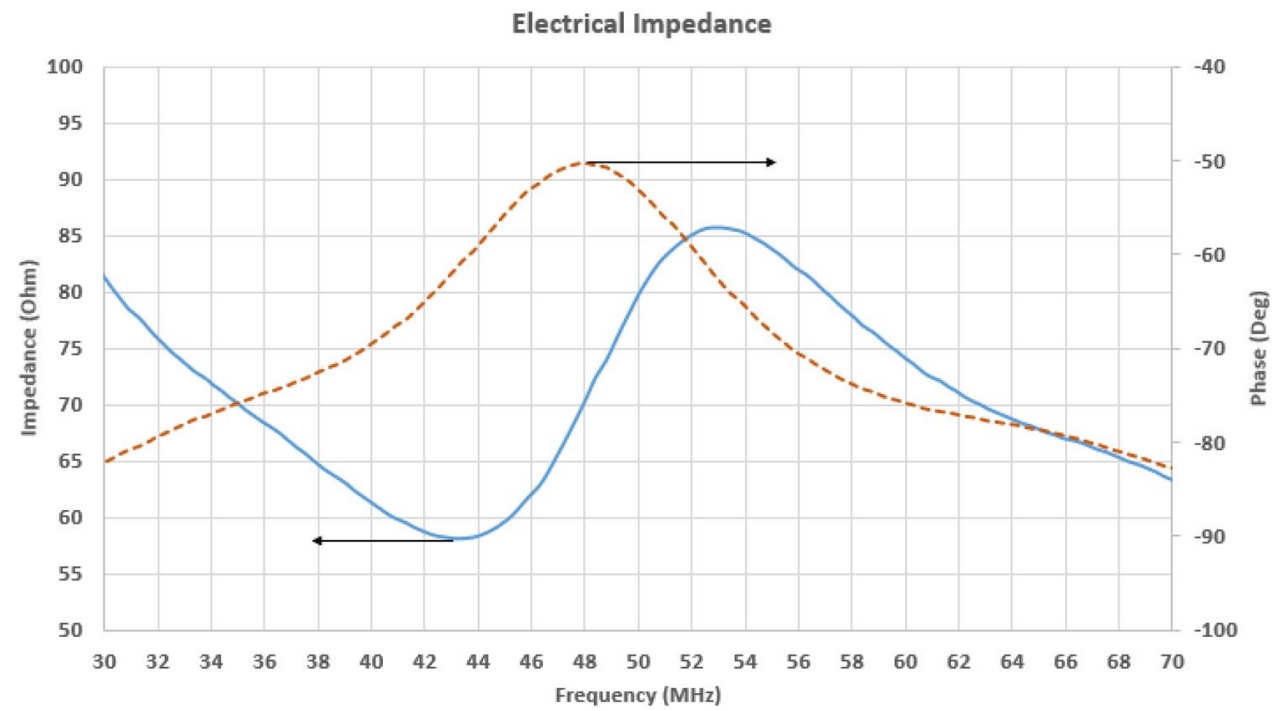

The Pressure resulting from impulse excitation

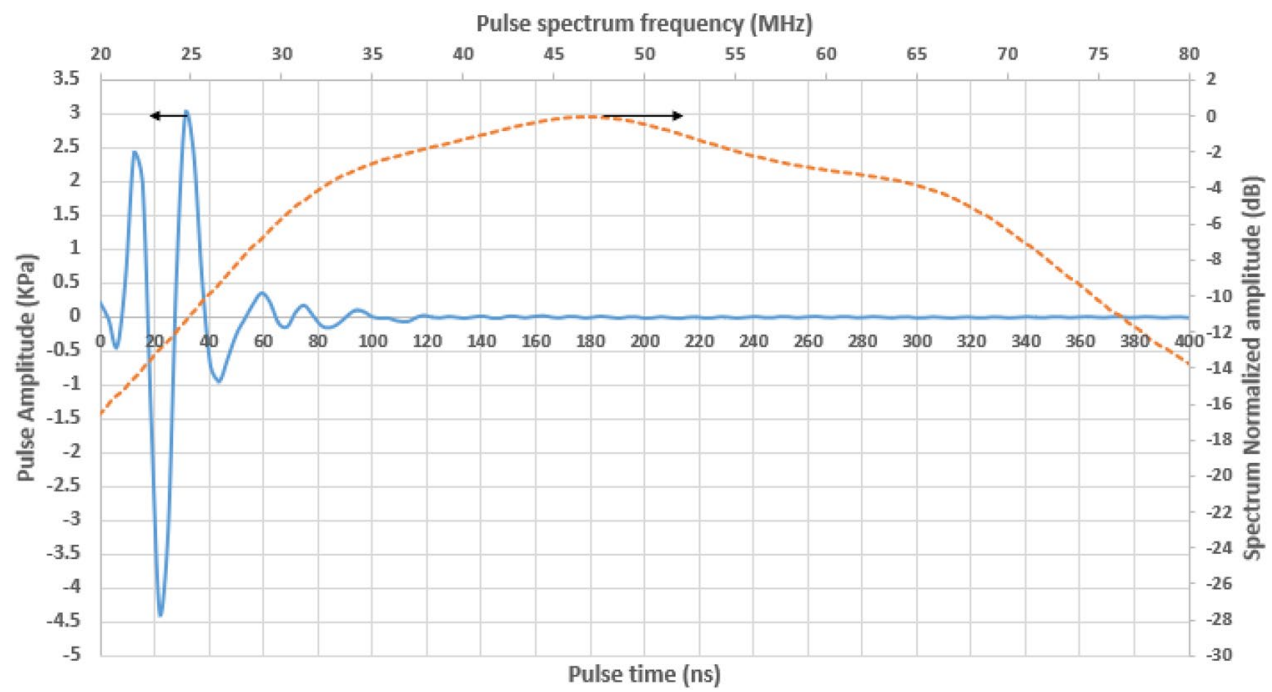

focal point can be seen in Fig. $6 \mathrm{~b}$. As it can be seen from Fig. $6 c$, which shows the dimensions of the image of the wire located at the exact focal point, the lateral resolution of the probe is $80 \mu \mathrm{m}$ and the axial resolution is $44 \mu \mathrm{m}$.
The thickness used in Table 3, unoptimized columns, has the optimal length for a single layer of material, but the complete probe layers are not necessarily optimal if all thicknesses follow this value. Also, the materials chosen 


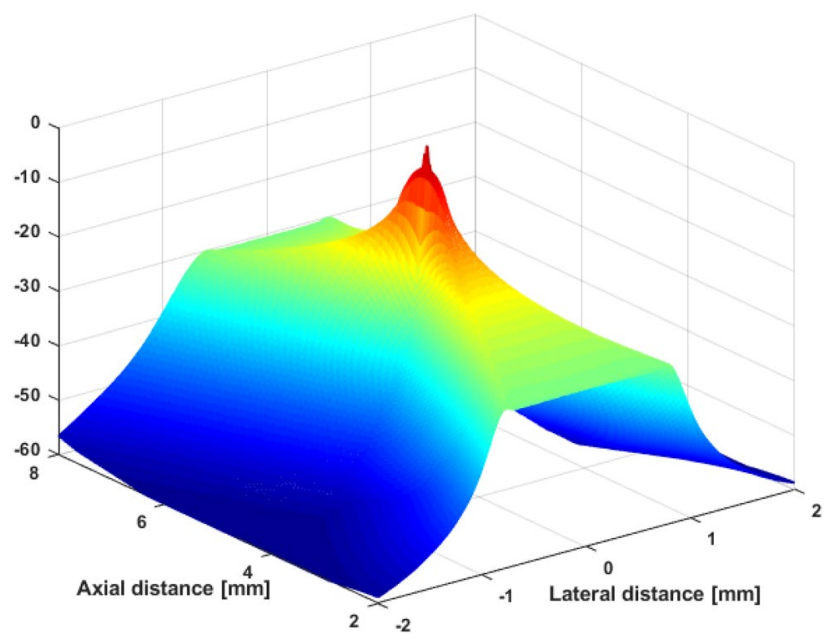

Fig. 5 The 3D profile of the simulated pressure field resulting from the probe. This profile was simulated using Field II

still have noticeable difference in acoustic impedance value (Z) with suggested values in Eqs. 6 and 7, so an optimization loop will be required if the best sound transmission or resulting imaging resolutions are intended.

After the proposed optimization algorithm, the best result can be seen in Fig. 7 and the corresponding thicknesses are shown in Table 3, columns related to the optimized transducer.

The resulting resolution of the optimized probe is 72 $\mu \mathrm{m}$ laterally and $42 \mu \mathrm{m}$ axially, which shows improvements over the conventionally designed transducer.
Looking at Fig. 8, which shows the pressure profile of the optimized transducer, it can be seen that the peakto-peak pressure has increased to $8.1 \mathrm{kPa}$ and the $-6 \mathrm{~dB}$ bandwidth of the pulse is $83 \%$ in the center frequency of $54 \mathrm{MHz}$. This shift in center frequency from the start frequency of $50 \mathrm{MHz}$ is due to the slight change in the thickness of the piezo-layer. This slight change has resulted in a better imaging resolution, so it is acceptable. Also, considering that after the manufacturing of the probe, its center frequency will change inevitably (due to manufacturing errors and material properties differences); $4 \mathrm{MHz}$ change in the center frequency of this probe does not cause a problem.

By simulating the pressure fields of the optimized and conventionally designed transducer (Fig. 9), it is clear that the optimized probe has a more focused and tight pressure field which leads to better imaging resolutions.

By comparing the conventionally designed and optimized results, it can be seen that the proposed algorithm can successfully find the best parameter values to obtain the finest imaging resolutions. Here, thicknesses of the layers were optimized and other parameters such as center frequency, head geometry, focal distance and material choices remained constant. Using the same procedure, the optimization problem can be solved for these parameters too. For example, one can use this method to find the best head geometry and center frequency for a desired resolution and depth of penetration. Considering that in the presented case, the frequency and the material choices have been predetermined, this result is the best possible

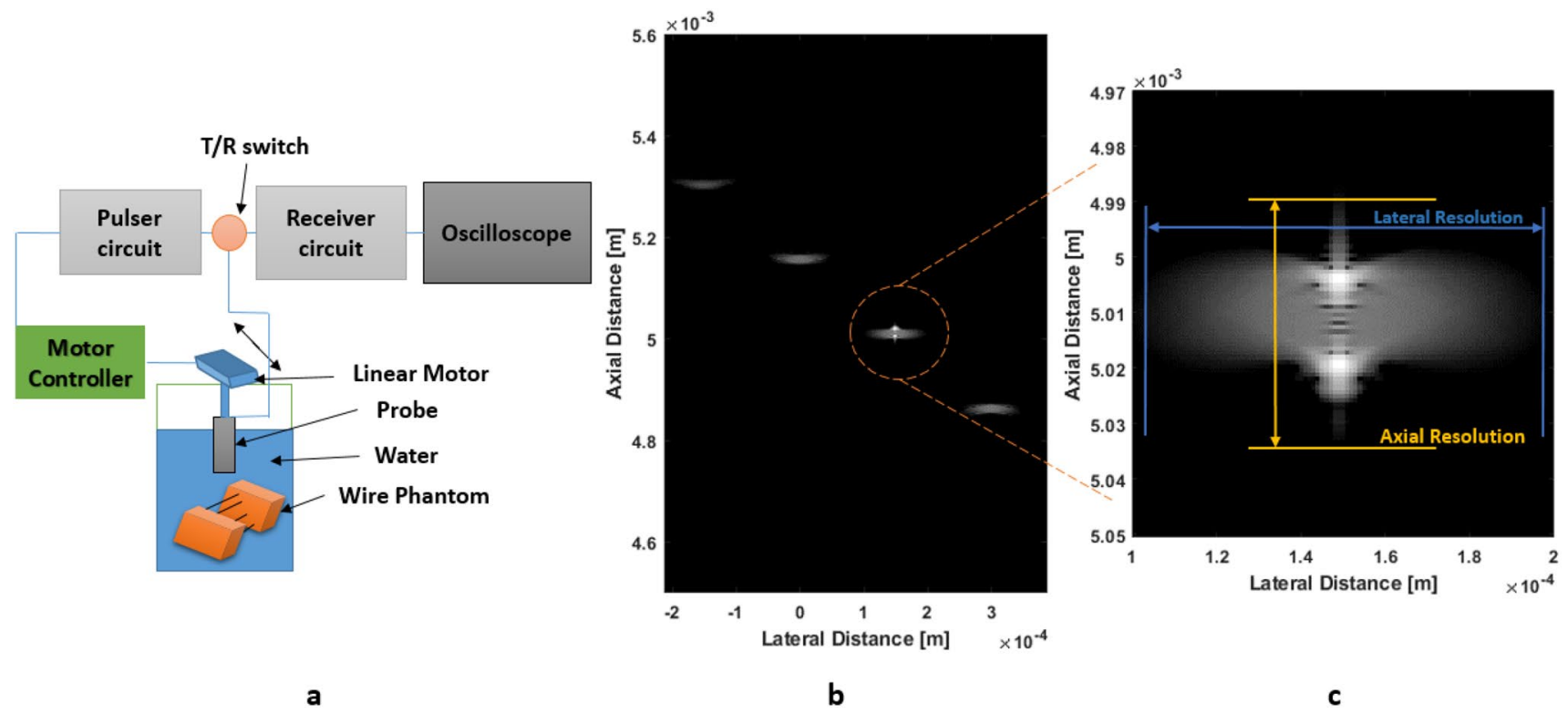

Fig. 6 Wire phantom imaging. a Schematics of the setup used for wire phantom imaging. b Simulated B-mode image of the wire. $\mathbf{c}$ Zoomed image of the wire located at the focal point. The dimen- sions used for determining the resolutions are shown. This simulation was performed using Field II

\section{SN Applied Sciences}



imaging of a wire placed in the focal point of the optimized probe with $40 \mathrm{~dB}$ dynamic range. The dimensions used for determining the resolutions are shown
Fig. 7 Simulated cross-section

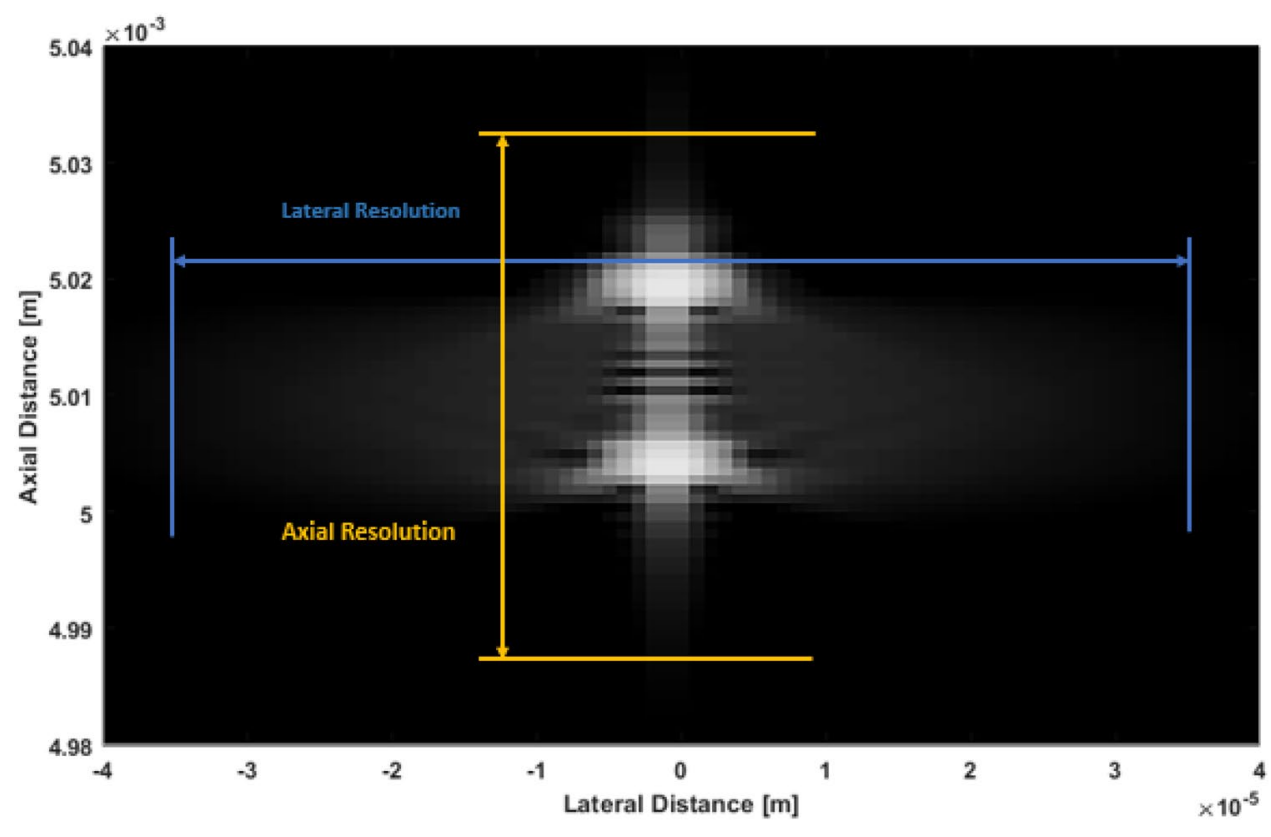

The Pressure resulting from impulse excitation

Fig. 8 The pressure profile in response to impulse voltage resulting from the KLM model for the optimized transducer. The red is the frequency spectrum of the signal

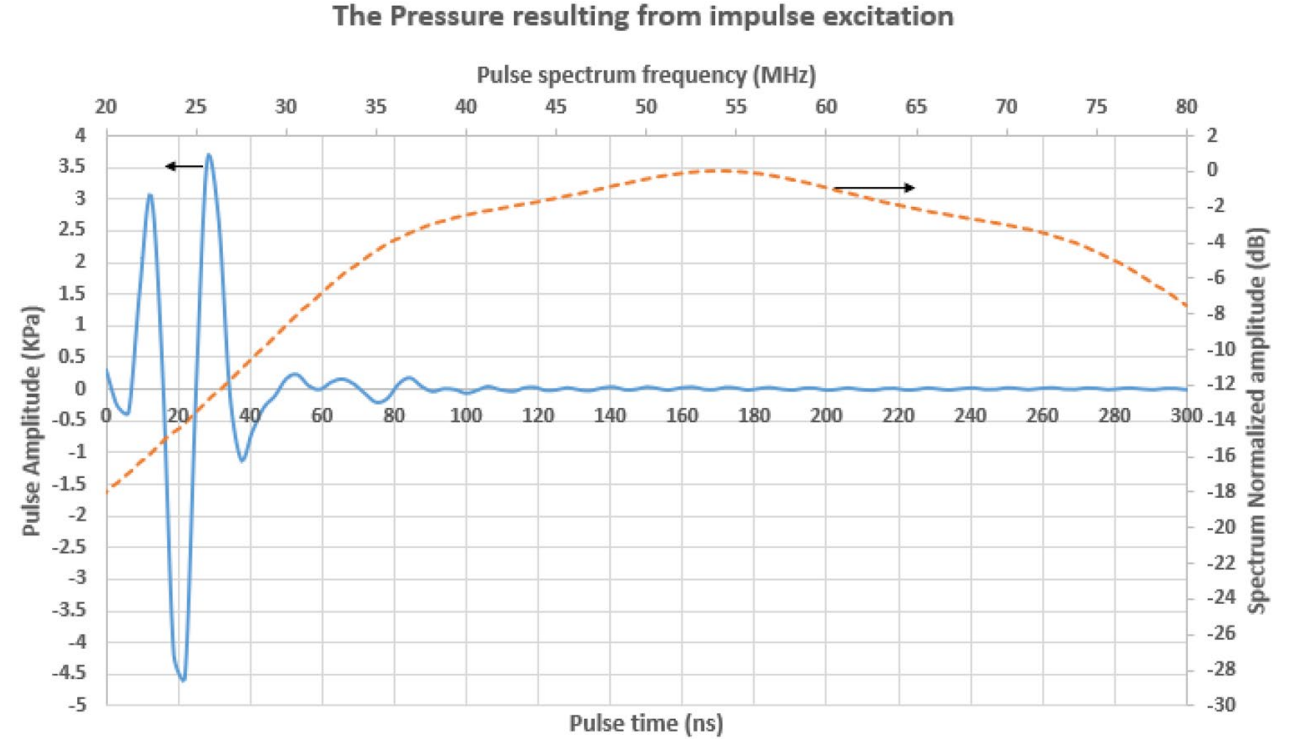

resolutions for this case, but if one uses a database of different materials and also different frequencies, it is possible to obtain better results using the described method.

The results prove that using the KLM model and Field II simulation together and creating an optimization loop, in which all of the transducer parameters are used to change the resulting pressure field and its characteristics to a desired field and values, is a powerful tool that can enable the automatic optimal design of ultrasound transducers for a specific pressure field and field characteristics.

It can be seen from the results that using the KLM model and Field II simulation together and creating an optimization loop in which all of the transducer parameters can be used to change the resulting pressure field and its characteristics to a desired field and values, is a novel approach, whereas the current design methods for high-frequency ultrasound transducers involve trial and error. As discussed in the paper, the novelty is to report a methodology that can be used to automate and optimize the previously mostly manual process of designing a transducer based on a given pressure field, which includes the intensity, the focal points and the transducer geometry to be built. To make such a pressure field, there are many parameters that can be changed such as layer thickness, center frequency, material choices, radius of the pressure field focusing of the head, etc. 

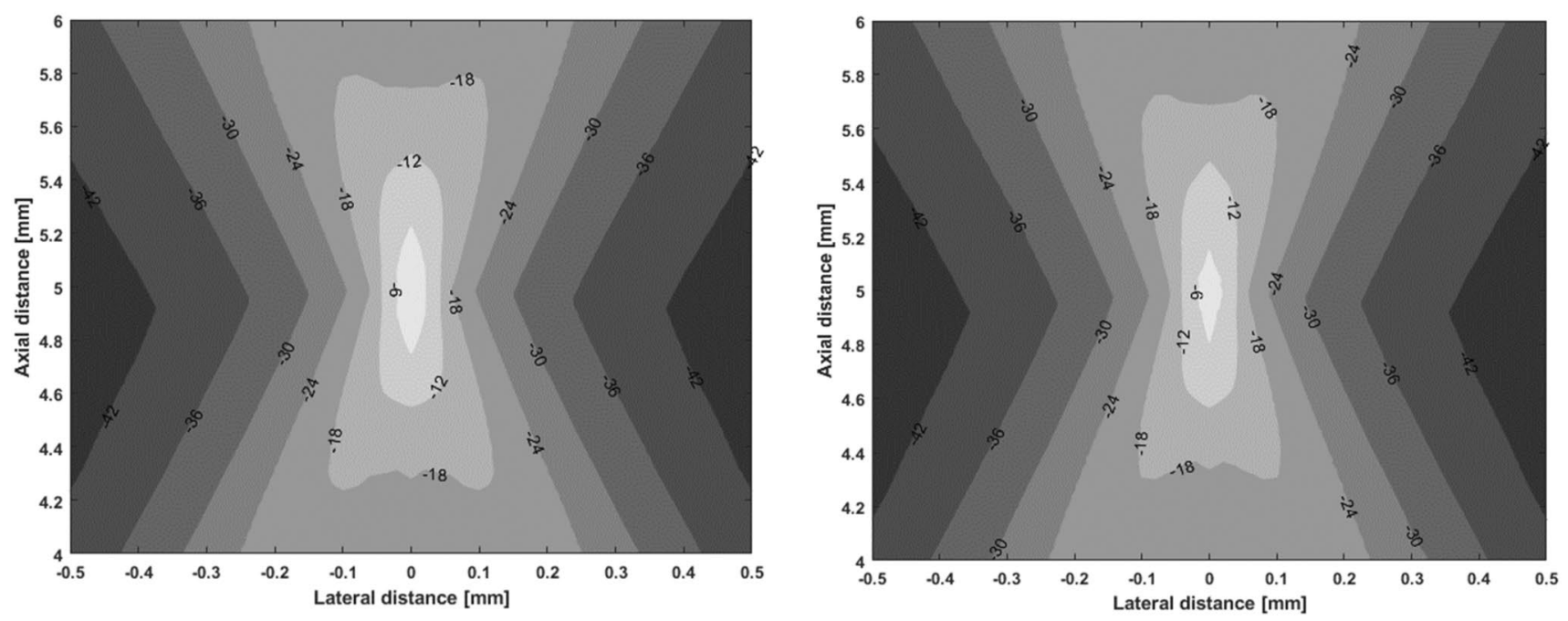

Fig. 9 Simulated pressure field contours (in dB) of the not optimized probe (left) and the optimized probe (right). This simulation is done using the Field II code

\section{Conclusion}

Theoretical consideration presented above has demonstrated that HF probes and their simulation methods can be optimized using GA and integration of KLM modeling and Field II as a pressure field calculating method. This method has been successfully implemented to optimize a probe design with the center frequency of around 50 $\mathrm{MHz}$, head diameter of $3 \mathrm{~mm}$ and a pressed focused focal length of $5 \mathrm{~mm}$. The optimization goal was to gain the finest lateral and axial imaging resolutions, which was simulated using the Field II source code, and its inputs were the thicknesses of the piezoelectric and matching layers. The proposed optimization loop can be directed to optimally design a HF medical US probe with the required imaging resolutions, impulse response bandwidth, focal distance, and material characterization.

\section{Declarations}

Conflict of interest The authors declare that they have no competing interests.

Open Access This article is licensed under a Creative Commons Attribution 4.0 International License, which permits use, sharing, adaptation, distribution and reproduction in any medium or format, as long as you give appropriate credit to the original author(s) and the source, provide a link to the Creative Commons licence, and indicate if changes were made. The images or other third party material in this article are included in the article's Creative Commons licence, unless indicated otherwise in a credit line to the material. If material is not included in the article's Creative Commons licence and your intended use is not permitted by statutory regulation or exceeds the permitted use, you will need to obtain permission directly from the copyright holder. To view a copy of this licence, visit http://creativecommons. org/licenses/by/4.0/.

\section{References}

1. Pavlin CJ, Foster FS (2012) Ultrasound biomicroscopy of the eye. Springer, Berlin

2. Kumagai K, Koike H, Nagaoka R et al (2012) High-resolution ultrasound imaging of human skin in vivo by using threedimensional ultrasound microscopy. Ultrasound Med Biol 38:1833-1838

3. Kozarova A, Kozar M, Minarikova E et al (2017) Identification of the age related skin changes using high-frequency ultrasound. Acta Medica Martiniana 17:15-20

4. Joodaki H, Panzer MB (2018) Skin mechanical properties and modeling: a review. Proc Inst Mech Eng Part H 232:323-343

5. Mundt GH, Hughes WF (2018) Ultrasonics in ocular diagnosis. Am J Ophthalmol 189:xxviii-xxxvi

6. Wang X, Seetohul V, Chen R et al (2017) Development of a mechanical scanning device with high-frequency ultrasound transducer for ultrasonic capsule endoscopy. IEEE Trans Med Imaging 36:1922-1929

7. Ma T, Yu M, Chen Z et al (2015) Multi-frequency intravascular ultrasound (IVUS) imaging. IEEE Trans Ultrason Ferroelectr Freq Control 62:97-107

8. Yoon S, Williams J, Kang BJ et al (2015) Angled-focused $45 \mathrm{MHz}$ PMN-PT single element transducer for intravascular ultrasound imaging. Sens Actuators A 228:16-22

9. Lee J, Moon J-Y, Chang J (2018) A 35 MHz/105 MHz dual-element focused transducer for intravascular ultrasound tissue imaging using the third harmonic. Sensors 18:2290

10. Latha S, Samiappan D, Kumar R (2020) Carotid artery ultrasound image analysis: a review of the literature. Proc Inst Mech Eng Part H 234(5):417-443 
11. Raj JR, Rahman S, Anand S (2016) An insight into elasticity analysis of common carotid artery using ultrasonography. Proc Inst Mech Eng Part H 230:750-760

12. Srinivasan S, Baldwin HS, Aristizabal O et al (1998) Noninvasive, in utero imaging of mouse embryonic heart development with 40-MHz echocardiography. Circulation 98:912-918

13. Yeo S, Yoon C, Lien C-L et al (2019) Monitoring of adult zebrafish heart regeneration using high-frequency ultrasound spectral doppler and Nakagami imaging. Sensors 19:4094

14. Shung KK, Zippuro M (1996) Ultrasonic transducers and arrays. IEEE Eng Med Biol Mag 15:20-30

15. Shinar Z, Chan L, Orlinsky M (2011) Use of ocular ultrasound for the evaluation of retinal detachment. J Emerg Med 40:53-57

16. de La Zerda A, Paulus YM, Teed R et al (2010) Photoacoustic ocular imaging. Opt Lett 35:270-272

17. Lee C, Lee J, Kim HH et al (2012) Microfluidic droplet sorting with a high frequency ultrasound beam. Lab Chip 12:2736-2742

18. Hsu H-S, Zheng F, Li Y et al (2012) Focused high frequency needle transducer for ultrasonic imaging and trapping. Appl Phys Lett 101:024105

19. Jeong JS, Lee JW, Lee CY et al (2011) Particle manipulation in a microfluidic channel using acoustic trap. Biomed Microdevice 13:779-788

20. Chen X, Lam KH, Chen R et al (2019) Acoustic levitation and manipulation by a high-frequency focused ring ultrasonic transducer. Appl Phys Lett 114:054103

21. Ohl S, Shrestha A, Khoo B et al (2010) Characterizing bubble dynamics created by high-intensity focused ultrasound for the delivery of antibacterial nanoparticles into a dental hard tissue. Proc Inst Mech Eng Part H 224:1285-1296

22. Moreau B, Vergari C, Gad H et al (2016) Non-invasive assessment of human multifidus muscle stiffness using ultrasound shear wave elastography: a feasibility study. Proc Inst Mech Eng Part H 230:809-814

23. van Velthoven $\mathrm{R}$, Aoun F, Marcelis $Q$ et al (2016) A prospective clinical trial of HIFU hemiablation for clinically localized prostate cancer. Prostate Cancer Prostatic Dis 19:79

24. Sklar LR, El Tal AK, Kerwin LY (2014) Use of transcutaneous ultrasound for lipolysis and skin tightening: a review. Aesthetic Plast Surg 38:429-441

25. Barthe PG, Slayton MH, Makin IRS (2019) Ultrasound probe for treating skin laxity. Google Patents, United States

26. Alshather $\mathrm{H}, \mathrm{Abd}$-Elsayed A (2019) Ultrasound in Chronic Pain Management. In: Abd-Elsayed A (ed) Pain. Springer, Cham. https://doi.org/10.1007/978-3-319-99124-5_50

27. Li J, Liu H, Li J et al (2019) Piezoelectric transducer design for an ultrasonic scalpel with enhanced dexterity for minimally invasive surgical robots. Proc Inst Mech Eng Part C 234:1271-1285

28. Shung KK (2015) Diagnostic ultrasound: Imaging and blood flow measurements. CRC Press, Boca Raton

29. Fei $C$, Chiu CT, Chen $X$ et al (2016) Ultrahigh frequency (100 $\mathrm{MHz}-300 \mathrm{MHz}$ ) ultrasonic transducers for optical resolution medical imagining. Sci Rep 6:28360

30. Foster FS, Pavlin CJ, Harasiewicz KA et al (2000) Advances in ultrasound biomicroscopy. Ultrasound Med Biol 26:1-27

31. Zhou Q, Lau S, Wu D et al (2011) Piezoelectric films for high frequency ultrasonic transducers in biomedical applications. Prog Mater Sci 56:139-174

32. Chabok HR (2011) Development of high frequency composites for ultrasound transducer applications. University of Southern California, Los Angeles

33. Zeng FC, Zhang XH (2012) Optimal Design of an ultrasonic transducer in rotary ultrasonic machining. In: Sung W-P, Kao J (C.M.), Chen R (eds) Applied mechanics and materials. Trans Tech Publ, pp 352-355. https://doi.org/10.4028/www.scientific.net/AMM. $184-185.352$
34. Zhang XH, Li GH (2010) Ultrasonic transducer design via APDL optimization. In: Li S, Liu Y, Zhu R, Li H, Ding W (eds) Applied mechanics and materials. Trans Tech Publ, pp 1076-1081. https://doi.org/10.4028/www.scientific.net/AMM.34-35.1076

35. Schröder A, Rautenberg J, Henning B (2010) Evaluation of cost functions for FEA based transducer optimization. Phys Procedia 3:1003-1009

36. Heikkola E, Miettinen K, Nieminen P (2006) Multiobjective optimization of an ultrasonic transducer using NIMBUS. Ultrasonics 44:368-380

37. Sadeghpour S, Puers R (2018) Optimization in the design and fabrication of a PZT piezoelectric micromachined ultrasound transducer (PMUT). In: Multidisciplinary digital publishing institute proceedings. $p 743$

38. Krimholtz R, Leedom DA, Matthaei GL (1970) New equivalent circuits for elementary piezoelectric transducers. Electron Lett 6:398-399

39. Jensen JA (1996) Field: a program for simulating ultrasound systems. In: 10th Nordicbaltic conference on biomedical imaging, vol 4, supplement 1, part 1:351-353, Citeseer

40. Lockwood GR, Foster FS (1994) Modeling and optimization of high-frequency ultrasound transducers. IEEE Trans Ultrason Ferroelectr Freq Control 41:225-230

41. He Z (2014) Optimization of acoustic emitted field of transducer array for ultrasound imaging. Bio-Med Mater Eng 24:1201-1208

42. Teresa Pietrzak A, Dybiec E, Adamczyk M et al (2015) High frequency ultrasonography of the skin and its role as an auxillary tool in diagnosis of benign and malignant cutaneous tumors-a comparison of two clinical cases. Acta Dermatovenerol Croat 23:43-43

43. Malvehy J, Pellacani G (2017) Dermoscopy, confocal microscopy and other non-invasive tools for the diagnosis of non-melanoma skin cancers and other skin conditions. Acta Derm Venereol. https://doi.org/10.2340/00015555-2720

44. Pasquali $P$ (2017) Ex vivo high-frequency ultrasound imaging of the skin. In: Humbert P, Fanian F, Maibach H, Agache P (eds) Agache's measuring the skin. Springer, Cham. https://doi.org/ 10.1007/978-3-319-32383-1_50

45. Bezugly A (2015) High frequency ultrasound study of skin tumors in dermatological and aesthetic practice. Med Ultrason 17:541-544

46. Cannata JM, Ritter TA, Chen W-H et al (2003) Design of efficient, broadband single-element $(20-80 \mathrm{MHz}$ ) ultrasonic transducers for medical imaging applications. IEEE Trans Ultrason Ferroelectr Freq Control 50:1548-1557

47. Foster FS, Lockwood G, Ryan L et al (1993) Principles and applications of ultrasound backscatter microscopy. IEEE Trans Ultrason Ferroelectr Freq Control 40:608-617

48. Levassort F, Tran-Huu-Hue L-P, Marechal P et al (2005) Characterisation of thin layers of parylene at high frequency using PZT thick film resonators. J Eur Ceram Soc 25:2985-2989

49. Kino GS (1987) Acoustic waves: devices, imaging, and analog signal processing Prentice-Hall Signal Processing Series. Prentice-Hall, Englewood Cliffs

50. Lockwood GR, Turnbull DH, Foster FS (1994) Fabrication of high frequency spherically shaped ceramic transducers. IEEE Trans Ultrason Ferroelectr Freq Control 41:231-235

51. Stepanishen PR (1971) The time-dependent force and radiation impedance on a piston in a rigid infinite planar baffle. J Acoust Soc Am 49:841-849

52. Stepanishen PR (1971) Transient radiation from pistons in an infinite planar baffle. J Acoust Soc Am 49:1629-1638

53. Penttinen A, Luukkala M (1976) The impulse response and pressure nearfield of a curved ultrasonic radiator. J Phys D Appl Phys 9:1547 
54. JrA J (1991) A model for the propagation and scattering of ultrasound in tissue. J Acoust Soc Am 89:182-190

55. Jensen JA, Svendsen NB (1992) Calculation of pressure fields from arbitrarily shaped, apodized, and excited ultrasound transducers. IEEE Trans Ultrason Ferroelectr Freq Control 39:262-267

56. Jensen JA, Gandhi D, WD Jr OB (1993) Ultrasound fields in an attenuating medium. In: 1993 Proceedings IEEE ultrasonics symposium 1993. IEEE, pp 943-946
57. Guo $P$, Wang $X$, Han $Y$ (2010) The enhanced genetic algorithms for the optimization design. In: 2010 3rd international conference on biomedical engineering and informatics 2010. IEEE, pp 2990-2994

Publisher's Note Springer Nature remains neutral with regard to jurisdictional claims in published maps and institutional affiliations. 